\title{
MICROBUNCHING AND BEAM BREAK-UP IN DUV-FEL ACCELERATOR
}

\author{
T. Shaftan ${ }^{\#}$, H.Loos, B. Sheehy, L. Carr, BNL, Upton, USA \\ Z. Huang, C. Limborg, SLAC, Menlo Park, USA, \\ W.S. Graves, MIT-Bates, Boston, USA
}

\begin{abstract}
We present the results of electron beam longitudinal modulation studies in the DUV-FEL accelerator. For bunch length determination we used the "zero-phasing" method, based on a measurement of the chirped electron bunch energy spectra. The measurements revealed a spiky structure in the longitudinal phase space [1]. A model based on space charge effect is considered [2] to explain of the obtained phenomena.

The analysis of the energy spectra has shown a sensitivity of the structure to the electron beam peak current, energy and longitudinal non-uniformity of the RF gun drive laser. Analytical calculations have demonstrated a qualitative agreement with experimental observations. Several experiments have been made to compare with theory; measured results are reviewed in this paper. The obtained effect is briefly discussed in relation to high brightness accelerators.
\end{abstract}

\section{INTRODUCTION AND MOTIVATION}

A detailed description of the DUV-FEL accelerator can be found in our earlier publications [3]. The DUV-FEL includes a photocathode RF gun, illuminated by a short pulse Ti:Sa laser. The following two linac sections accelerate the beam up to an energy of $70 \mathrm{MeV}$. The second linac tank is dephased, producing a time-energy correlation, which a four-magnet chicane, located downstream, converts into longitudinal bunching. Since the bunch is "undercompressed", the third linac tank is used to remove residual chirp, and, in combination with the fourth tank, accelerates the bunch up to the nominal energy for FEL operations (177 MeV).

For a successful FEL performance a peak current of 300 $\mathrm{A}$ is needed, which requires an initial bunch length of 1.5 ps RMS for the $300 \mathrm{pC}$ electron bunch to be reduced to 0.4 ps RMS (compression ratio of 3.75).

For the electron bunch length characterization we use the "zero-phasing" method [4], in which no further acceleration is done after the bunch compressor, but an energy chirp is imparted to the beam with the last linac section (with calibrated RF amplitude). A $72^{\circ}$ spectrometer dipole transforms the correlated energy spread into a spatial distribution on a downstream monitor. The RMS size of the horizontal projection $\sigma_{x}$, due to a known amount of chirp $\mathrm{E}_{\mathrm{RF}} \cdot \mathrm{k}_{\mathrm{RF}}$, corresponds to the bunch length $\sigma_{z}$ :

$$
\sigma_{z}=\frac{E_{0}}{E_{R F}} \frac{1}{\eta \cdot k_{R F}} \sigma_{x},
$$

where $\eta$ is the dispersion and $E_{0}$ the energy at the monitor location.
First measurements of the compressed bunch length demonstrated a strong modulation of the energy spectra, obtained via "zero-phasing" [1]. In some cases the electron beam image became broken into several distinctive bunches with a separation of the order of 200 fs (Fig. 1). At the same time the uncompressed bunch spectra were smooth, witnessing the existence of a collective effect, which is driving the modulation during the compression process. For low charge $(\sim 20 \mathrm{pC})$ spikes are visible and sharp even for an uncompressed beam.

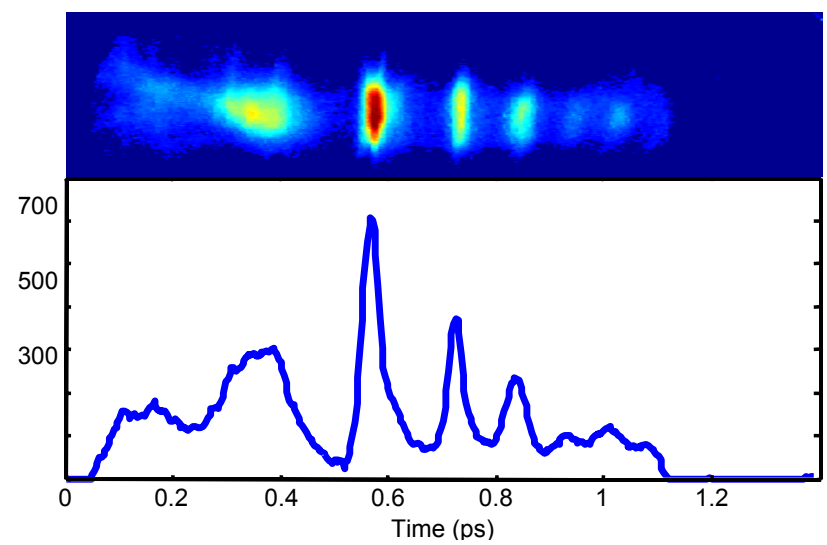

Figure 1: Modulated beam image after spectrometer (upper figure), vertical axes is y-coordinate; horizontal one is proportional to beam energy. Bottom figure: "zerophasing" projection of the beam on upper plot, horizontal axis is scaled in picoseconds.

Since the chirping tank is running at zero-crossing, it does not introduce a nonlinearity into the longitudinal phase space. The spectrometer magnet aberrations are also small, so the assumption about a linear correspondence between the chirped bunch energy spectrum and the longitudinal bunch density seems to be valid if no collective effects are taken into account. Therefore the sharp spikes in the electron energy spectra could be treated as spikes in the longitudinal bunch density. Calculating the FEL slippage length as 70 um for lasing at $266 \mathrm{~nm}$, it follows that the spike width was comparable or less than the slippage length, which had to cause a degradation of the FEL performance. Thus the role of the collective effect has to be studied, also considering that a similar effect has been obtained recently in the TESLA linac [5].

\section{INITIAL EXPERIMENTS AND OBSERVATIONS}

We observed strong modulations (as large as $100 \%$ ) in the "zero-phasing" projection for the uncompressed bunch at low charge $(20 \mathrm{pC})$. The fundamental harmonic of the 
modulation frequency spectrum peaks at $1.6 \mathrm{THz}(190$ $\mu \mathrm{m})$. This high frequency range can be associated only with the drive laser (266 nm, bandwidth 5\% RMS), since the RF bandwidth in all other accelerator components is limited to several $\mathrm{GHz}$. We measure the temporal profile of the laser by cross correlating it with the $120 \mathrm{fsec}, 800$ $\mathrm{nm}$ oscillator pulse in a nonlinear optical crystal. This measurement has a resolution of $\sim 200$ fs, due to velocity mismatch in the crystal. Some modulation is observed (Fig. 2). The magnitude of the modulation may be larger than indicated in the figure if its period is below the crosscorrelator's resolution, or due to the fact that the crosscorrelation is a multi-shot measurement.

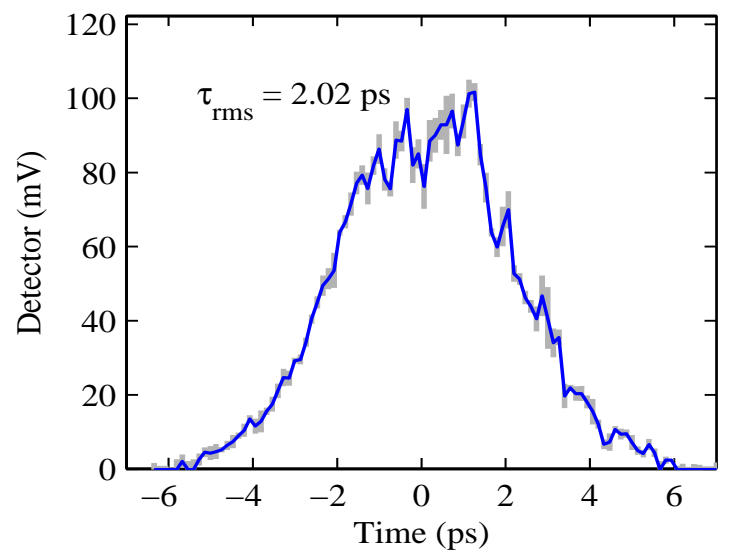

Figure 2: Laser cross-correlation profile.

For an explanation of the modulation dynamics for the uncompressed bunch we developed the following hypothesis. The initial laser modulation gets mapped into the electron bunch phase space at the low energy end of the accelerator (in the gun and first linac tank). At low charge the space charge forces transfer initial density nonuniformities into an energy modulation along the bunch. Since the uncorrelated energy spread is small $\left(\sigma_{\gamma} / \gamma \sim 10^{-5}\right)$, an energy modulation $\left(\sim 10^{-4}\right.$ for $\left.20 \mathrm{pC}\right)$ becomes visible in the energy spectrum of the chirped bunch.

For an uncompressed bunch with higher charge the space charge forces become stronger and smear out the energy modulation. This increases the projected beam energy spread and reduces the observed modulation in the chirped beam energy spectrum.

This hypothesis is currently being studied using the PARMELA code.

Since the modulation is sensitive to the peak current and appears in an initially smooth beam during the compression process, one should consider a collective effect, responsible for the development of structure. Earlier [1], we discussed CSR in the chicane as a possible mechanism for the instability. However, it has been shown [6] that the effect of CSR leads only to a small gain for microbunching. Another possible source of microbunching is the spectrometer dipole, which itself has a strong impact on the longitudinal beam dynamics of the electron beam. Calculations of CSR and space charge effects in the spectrometer dipole [2] exhibited very low gain for this case as well.
A CSR-mediated effect has to be sensitive to the bending radius in the chicane magnets. We compressed the beam for several different chicane strengths $\left(\mathrm{R}_{56}\right)$, keeping the final bunch length constant. Since the compression ratio is equal to $1-\mathrm{h} \mathrm{R}_{56}$, where $\mathrm{h}$ is the energy chirp, there is always a combination of $h$ and $R_{56}$ that will maintain a constant compression ratio and, therefore, a constant final peak current of the bunch. Thus the initial and final beam properties do not change* for different chicane strengths and we can distinguish whether the modulation is sensitive to the bending radius of the chicane or not. The result of this experiment is that, though there is some reduction of modulation for smaller $\mathrm{R}_{56}$ at low compression ratio, in general, the modulation is insensitive to chicane settings.

\section{MODEL OF THE EFFECT AND COMPARISON WITH EXPERIMENT}

It has been shown [2], that the space charge force for our experimental conditions can be strong enough to transform small longitudinal density non-uniformities into a modulation of the average energy along the bunch. The plasma oscillation wavelength of the compressed beam becomes comparable to the length of the drift between chicane and spectrometer $(\sim 15 \mathrm{~m})$ for the wavelength range of the modulation. Chirping such an energymodulated bunch (Fig. 3) one would see sharp spikes in the energy spectrum, corresponding to variations of the energy. At the same time this effect cannot enhance the density bunching in the electron beam, since the longitudinal space charge force is equivalent to a pure imaginary capacitive impedance.

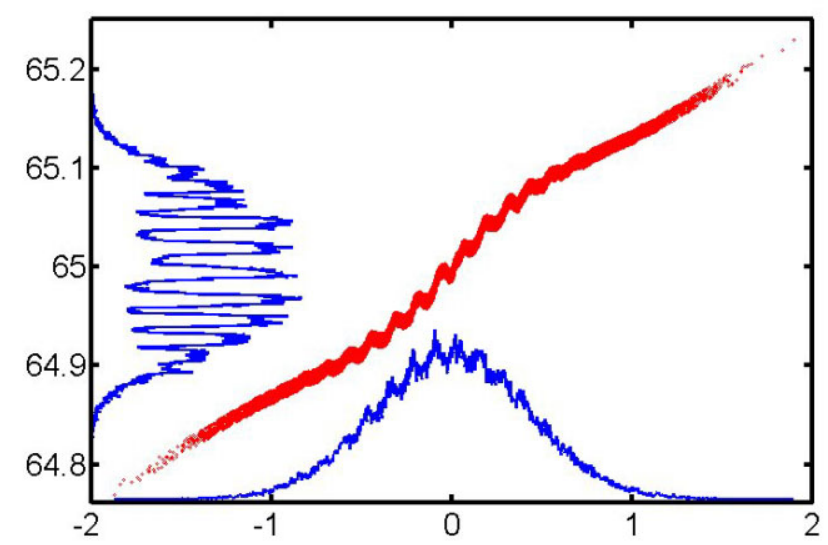

Figure 3: Illustration of electron bunch longitudinal phase space. Abscissa is time (ps), ordinate is energy $(\mathrm{MeV})$. The space charge wake induces variations of the energy along the chirped bunch leading to deeply modulated energy spectra.

As the space charge impedance depends only on the beam energy and transverse size (assuming uniform

\footnotetext{
* Note, also, that the transverse beam envelope along the accelerator has been kept constant during measurements.
} 
transverse distribution and no vacuum chamber), we performed experiments, varying these beam parameters and studying the modulation.

Accelerating or decelerating the compressed beam in the third linac tank we varied the energy of the electrons from 50 to $110 \mathrm{MeV}$. Zero-phasing projections of the electron beam for different energies show that the modulation reduces with increasing energy, as expected.

Using three lattices with different beam envelopes $(0.5$, $1.0,1.5 \mathrm{~mm}$ RMS) we measured the dependence of the modulation versus beam size. It was explicitly shown, that the modulation, being present when the beam size is small, almost vanishes at a larger beam cross section.

Measuring the dependence of the modulation versus charge was an important stage in the experiments. Electron beams with different charge $(40,100,200,300$ pC) were generated, compressed and transported to the spectrometer dipole. Special attention was paid to insure both the transverse and longitudinal particle transport along the accelerator to be approximately the same for different amounts of bunch charge. In this case only the peak current was scaled together with changing the beam charge.

The measurements done for the uncompressed beam revealed the dynamics of the structure at low charge, where the modulation is strong and visible, but only weakly depends on the particle transport at high energy. During the compression the modulation wavelength gets scaled down according to the compression ratio without a significant enhancement of the structure.

As the peak current in the uncompressed beam increases the structure vanishes, and the beam exhibits an almost smooth distribution for some $300 \mathrm{pC}$ of charge. After compression the modulation reappears, but the period of modulation stays the same as for the case of low charge.

Finally we checked if the beam modulation is associated with bunching in the longitudinal particle density. Varying the electron beam size along the channel we generated "modulated" and "non-modulated" beams. Using an IR bolometer together with low-pass IR filters (cut-off at $40 \mu \mathrm{m}, 100 \mu \mathrm{m}, 160 \mu \mathrm{m}$ ) we measured CTR from a metallic mirror, installed inside the vacuum chamber. The characteristic modulation wavelength was estimated as $90 \mu \mathrm{m}$, using chirped bunch profiles. Measuring the CTR power with different filters we have not obtained any difference between "modulated" and "non-modulated" beam conditions. This proves that the modulation does not introduce any additional density bunching in the electron beam.

\section{CONCLUSION}

In this paper we discussed the peculiar effect of longitudinal beam dynamics in the DUV-FEL linear accelerator. A space charge model of the phenomenon is in qualitative agreement with experimental data. The experiments show a sensitivity of the effect to the electron beam energy, transverse size and peak current. It was also shown that the induced modulation does not enhance the density bunching in the electron beam. Since the drive laser is a likely source of this structure, high-resolution optical diagnostics are needed for more quantitative studies. Therefore a single-shot SPIDER apparatus [7] with high resolution is being developed.

One of the important questions is whether this structure can be dangerous for the accelerator performance. First, this effect is sensitive to the initial laser structure, so for a perfectly uniform longitudinal laser profile a modulation does not exist, unless it is created on latter stages in the accelerator. At the same time this structure, if present in the beam, can cause at least two problems. First, it increases the projected energy spread in the bunch. Second, the modulation, developed in the beam at low energy, becomes "frozen" at a higher energy. Then, any magnetic system (bending system, chicane-compressor) may convert this modulation into real spatial bunching for a modulation wavelength of $\lambda_{\bmod }=2 \mathrm{R}_{56} \Delta \gamma_{\bmod } / \gamma$, where $\Delta \gamma_{\text {mod }}$ is the amplitude of the space charge induced energy spread. Now this effect can degrade the electron beam quality due to coherent radiation of the spikes during the compression process. So, for any specific configuration of the accelerator, the allowable amount of modulation must be calculated and the appropriate laser quality established.

\section{ACKNOWLEDGEMENTS}

We would like to thank D. Dowell, A. Doyuran, P. Emma, S. Krinsky, J.B. Murphy, J. Rose, X.J. Wang, J. $\mathrm{Wu}, \mathrm{Z} . \mathrm{Wu}, \mathrm{L} . \mathrm{H}$. Yu for many useful discussions and comments. This work is performed under DOE contract DE-AC02-76CH00016.

\section{REFERENCES}

[1] "Ultrashort electron bunch length measurements at DUVFEL", W. Graves et al., PAC 2001, Chicago, p. 2224 [2] "Beam energy modulation and its impact on rf zerophasing bunch profile measurement", Z. Huang, SLACPUB-9788

[3] "Electron Bunch Compression in the SDL linac", T.Shaftan et al., EPAC'02, Paris, June 2002,p. 834.

[4] "Measurements of femtosecond electron bunches using a rf zero-phasing method", D.X. Wang et. al., Phys. Rev., E, 57, 2283 (1998)

[5] "Observation of longitudinal phase space fragmentation at the TESLA test facility free-electron laser", Ph. Piot et al., NIM A 475 (2001), pp. 348-352

[6] "Experiments in Coherent Radiation at SDL", H. Loos et al., EPAC'02, Paris, June 2002,p. 814.

[7] "Spectral phase interferometry for Direct ElectricField Reconstruction of Ultrashort Optical Pulses", C. Iaconis et al., Opt. Lett. 23, 792 (1998).

\# Corresponding author, e-mail: shaftan@bnl.gov 